\title{
Compound sums and subexponentiality
}

\author{
HANSPETER SCHMIDLI \\ Department of Theoretical Statistics, University of Aarhus, Ny Munkegade, 8000 Aarhus C, \\ Denmark.E-mail: schmidli@imf.au.dk
}

We investigate compound distributions - for example, compound mixed Poisson distributions - in the case where the summands, the mixing distribution or the number of summands are subexponential. It is shown that such distributions are subexponential. As an illustration the tail of the maximum of a Björk-Grandell process is obtained.

Keywords: compound distribution; extreme-value theory; integrated tail distribution; mixed Poisson distribution; subexponential distribution

\section{Introduction and main results}

In problems considered in applied probability - for example, risk theory, queuing theory or storage theory - the class $\mathscr{S}$ of subexponential distributions is quite important. In queuing theory heavy tails appear as a consequence of long-range dependence, and in insurance aggregate claims from catastrophe insurance have a heavy tail behaviour. $\mathscr{S}$, introduced by Chistyakov (1964), consists of all distributions $G$ with the property that

$$
\lim _{x \rightarrow \infty} \frac{\overline{G^{* 2}}(x)}{\bar{G}(x)}=2,
$$

where $\bar{G}(x)=1-G(x)$. Here $F * G$ denotes the convolution of the distribution functions $F$ and $G, G^{* 1}=G$ and $G^{*(n+1)}=G^{* n} * G$. The intuitive interpretation of this definition is that a sum of two independent random variables can only exceed a large threshold $x$ if one of the random variables exceeds the threshold $x$. A special class of subexponential distributions is $\mathscr{R}$, the class of distributions with a regularly varying tail, i.e.

$$
\lim _{x \rightarrow \infty} \frac{\bar{G}(x t)}{\bar{G}(x)}=t^{-\alpha}
$$

for some $\alpha \geqslant 0$. We will use the notation $X \in \mathscr{S}$ for a random variable $X$ if the distribution function $G$ of $X$ is subexponential.

In applications one often has to deal with compound sums $S_{N}=\sum_{i=1}^{N} Y_{i}$, where $N$ is a random variable taking values in $\mathbb{N}$ and $\left(Y_{i}\right)$ is a sequence of independent identically distributed positive random variables independent of $N$. The case where $N$ has a mixed Poisson distribution is of special interest; see, for instance, Embrechts et al. (1993), Grandell (1991) or Schmidli (1996). If $Y_{i} \in \mathscr{B}, N \in \mathscr{B}$ or the mixing distribution in the 
compound Poisson case is in $\mathscr{B}$ then it is possible to show that $S_{N} \in \mathscr{R}$; see the discussion following Theorem 1 below.

In recent work Asmussen et al. (1999a) show that in order to find the maximum of a process in the presence of heavy tails it is often enough only to consider the process at some regeneration points. The maximum of a process can be considered as the ruin probability in insurance risk or as the tail of the steady-state waiting time in a queuing model. The increment between two regeneration points then contains a term of the form of $S_{N}$. It is therefore of interest to know whether $S_{N} \in \mathscr{S}$ or not also in the case where $N \notin \mathscr{R}$. We will give an answer under a light-tail assumption on the other distributions involved. Another motivation for considering $N \in \mathscr{S}$ comes from hurricane insurance. Here the individual claims are bounded, i.e. light-tailed. But the number of claims makes the aggregate claims from one hurricane clearly show heavy tails. This indicates that $N$ is heavy-tailed.

We denote by $\Gamma$ the class of distributions $G$ with the property that either $G\left(x_{0}\right)=1$ for some $x_{0} \in(0, \infty)$ or

$$
\varliminf_{x \rightarrow \infty} \frac{\overline{G^{*(m+1)}}(x)}{\overline{G^{* m}}(x)} \geqslant a
$$

for some $a>1$ and all $m \in \mathbb{N}$. Note that for any $n \in \mathbb{N}$ condition (1) implies that

$$
\varliminf_{x \rightarrow \infty} \frac{\overline{G^{*(m+n)}}(x)}{\overline{G^{* m}}(x)} \geqslant a^{n} .
$$

All light-tailed distribution functions of practical interest belong to $\Gamma$. For instance, if $G$ has a gamma tail, i.e. $\bar{G}(x) \sim c x^{\gamma-1} \mathrm{e}^{-\alpha x}(\gamma \geqslant 0)$ then $G \in \Gamma$ by the lemma below. The class $\mathscr{S}(\alpha)$ of Embrechts and Goldie (1982) belongs to $\Gamma$ for $\alpha>0$, but $\mathscr{S}=\mathscr{S}(0)$ does not. Indeed, because $\overline{G^{* n}}(x) \sim n \bar{G}(x)$ (Lemma 2(iii)) it follows that $a \leqslant(n+1) / n$ and therefore $a \leqslant 1$. For a definition of $\mathscr{S}(\alpha)$, see Embrechts and Goldie (1982). Note that if $\bar{G}(x) \sim c x^{\gamma-1} \mathrm{e}^{-\alpha x}$ for some $\gamma<0$, then $G \in \mathscr{S}(\alpha)$.

The following condition is easier to verify than (1).

Lemma 1. Let $G$ be a distribution function such that $G(x)<1$ for all $x>0$ and

$$
\lim _{x \rightarrow \infty} \frac{\overline{G^{* 2}}(x)}{\bar{G}(x)}=\infty \quad \text { and } \quad \lim _{x \rightarrow \infty} \frac{\overline{G^{* 2}}(x)}{\bar{G}\left(x-x_{0}\right)} \geqslant \frac{1}{b}
$$

for all $x_{0}>0$ and some $b<1$ independent of $x_{0}$. Then $G \in \Gamma$.

Let us denote by $G$ the distribution function of the summands $Y_{i}$, by $F$ the distribution function of the sum $S_{N}=\sum_{i=1}^{N} Y_{i}$, and by $p_{n}=P[N=n]$ the distribution of the number $N$. We will henceforth use indices to denote independent copies of a random variable, for instance $N_{1}$ and $N_{2}$ will be independent random variables with $P\left[N_{i}=n\right]=p_{n}$. For notational convenience we deal with positive summands only, i.e. $G(0)=0$. Theorems 1 and 
2 below will also hold for the more general subexponential distribution functions with $G(0) \neq 0$.

We say $N$ has a mixed Poisson distribution with mixing distribution $H$ if

$$
p_{n}=\int_{0}^{\infty} \frac{\ell^{n}}{n !} \mathrm{e}^{-\ell} \mathrm{d} H(\ell),
$$

where $H$ will always denote the mixing distribution and has the property that $H(0-)=0$. Then we obtain the following result. The first part can be found in Grandell (1997); see also Athreya and Ney (1972, p. 150).

\section{Theorem 1.}

(i) Assume that there exists an $\varepsilon>0$ such that $\mathrm{E}\left[(1+\varepsilon)^{N}\right]<\infty$. If $Y \in \mathscr{S}$ then $S_{N} \in \mathscr{S}$. Moreover, $P\left[S_{N}>x\right] \sim \mathrm{E}[N] P[Y>x]$.

(ii) Assume that $G \in \Gamma$. If $N \in \mathscr{S}$ then $S_{N} \in \mathscr{S}$.

Corollary 1. Let $N$ have a mixed Poisson distribution. If $H \in \mathscr{S}$ then $N \in \mathscr{S}$. If, moreover, $G \in \Gamma$ then $S_{N} \in \mathscr{S}$.

Note that (i) is fulfilled if and only if $\int_{0}^{\infty} \mathrm{e}^{\varepsilon \ell} \mathrm{d} H(\ell)<\infty$ in a mixed Poisson model. It may be possible to extend Corollary 1 to other mixed distributions. In applications, however, the mixed Poisson distribution is of special interest as a generalization of classical models - for instance, the Cramér-Lundberg model in risk theory or the $\mathrm{M} / \mathrm{G} / 1$ queuing model, where $N$ has a Poisson distribution.

In the regularly varying case the assumptions of Theorem 1 can be weakened and the explicit asymptotic behaviour can be obtained. The following case was proved in Stam (1973). Let $L(x)$ be a slowly varying function, i.e. $L(x t) / L(x) \rightarrow 1$ as $x \rightarrow \infty$. Assume

$$
\lim _{x \rightarrow \infty} L(x) x^{\alpha} \bar{G}(x)=\beta, \quad \lim _{n \rightarrow \infty} L(n) n^{\alpha} P[N>n]=\gamma,
$$

for some $\beta, \gamma \in[0, \infty)$. If $\mathrm{E}[Y], \mathrm{E}[N]<\infty$ (this implies $\alpha \geqslant 1$ ), or if $0 \leqslant \alpha<1$ and $\mathrm{E}[N]<\infty$ (this implies $\gamma=0$ ), or if $0 \leqslant \alpha<1$ and $\mathrm{E}[Y]<\infty$ (this implies $\beta=0$ ), then

$$
\lim _{x \rightarrow \infty} L(x) x^{\alpha} \bar{F}(x)=\gamma \mathrm{E}[Y]^{\alpha}+\beta \mathrm{E}[N] .
$$

If the tail of the distribution of $N$ is thicker than the tail of the distribution of $Y$, as it is the case in the situation of Theorem 1(ii), we have in the case $N \in \mathscr{R}$

$$
P\left[S_{N}>x\right] \sim \gamma(x / \mathrm{E}[Y])^{-\alpha} / L(x) \sim \gamma(x / \mathrm{E}[Y])^{-\alpha} / L(x / \mathrm{E}[Y]) \sim P[N>x / \mathrm{E}[Y]] .
$$

This result tells us that $S_{N}$ only can become large if $N$ becomes large, and that, conditioned on $S_{N}>x$, the conditional mean of $Y_{i}$ is asymptotically $\mathrm{E}[Y]$. Indeed, for a large $N$ the strong law of large number implies $S_{N} / N \approx \mathrm{E}\left[Y_{i} \mid S_{N}>x\right]$ given $S_{N}>x$. Grandell (1997, Proposition 8.4 and Corollary 8.5) shows that, for $\alpha \neq 1, L(n) n^{\alpha} P[N>n] \rightarrow \gamma$ as $n \rightarrow \infty$ holds if $N$ is mixed Poisson distributed with a mixing distribution $H$ satisfying $\bar{H}(\ell) L(\ell) \ell^{\alpha} \rightarrow \gamma$ as $\ell \rightarrow \infty$, i.e. $P[N>n] \sim \bar{H}(n)$.

It seems natural to expect $P\left[S_{N}>x\right] \sim P[N>x / \mathrm{E}[Y]]$ also in the case $N \in \mathscr{S}$ or 
$P[N>n] \sim \bar{H}(n)$ also in the case $H \in \mathscr{S}$. But, as often happens for subexponential distributions, intuition fails, as the following counterexample shows.

Let $\tilde{N}$ be Weibull distributed, i.e. $P[\tilde{N}>x]=\exp \left\{-x^{\alpha}\right\}$, and let $N=[\tilde{N}]$ denote the integer part of $\tilde{N}$. Assume $\frac{1}{2}<\alpha<1$. Then $N$ is subexponential. Let $Y$ be Poisson distributed with mean 1. It is easy to see that $Y$ fulfils (3). From Theorem 1 it follows that $S_{N} \in \mathscr{S}$. Let $(X(t))$ be a Poisson process with rate 1 . Note that $S_{N}$ and $X(N)$ have the same distribution. From Lemma 2(vii) we find that $\bar{F}(x) \sim P[X(\tilde{N})>x]$. Note that $X(\tilde{N})$ has a mixed Poisson distribution, and we also obtain a counterexample to the conjecture $P[X(\tilde{N})>n] \sim P[\tilde{N}>n]$. It is shown in Asmussen et al. (1999b) that

$$
P[X(\tilde{N})>x] \sim \exp \left\{-x^{\alpha}\left(1-\left(\alpha+\frac{x^{1-\alpha}}{\alpha}\right)^{-1}\right)^{\alpha}-\alpha x^{\alpha}-1+\frac{x}{\alpha+x^{1-\alpha} / \alpha}\right\} .
$$

Thus the tail of $S_{N}$ is heavier than the tail of $N$. It seems that the Weibull distribution with $\alpha>\frac{1}{2}$ is not 'heavy-tailed enough' for $N$ to be the 'only' variable responsible for $S_{N}>x$. As in the light-tail case $S_{N}$ can only become large if both $N$ and the $Y_{i}$ are large.

In applications one is often interested in the probability that a random walk with negative drift exceeds a certain threshold. This probability is called the ruin probability in risk theory and the tail of the steady-state waiting time distribution in queuing theory. For this problem it is not the distribution of $Y$ itself that is important but the integrated tail distribution defined by

$$
G_{0}(x)=\frac{1}{\mathrm{E}[Y]} \int_{0}^{x} P[Y>y] \mathrm{d} y
$$

see Veraverbeke (1977) or Embrechts and Veraverbeke (1982). If we, for example, consider the random walk with increments $Y_{i}-T_{i}$, where $\left(T_{i}\right)$ is a sequence of positive independent and identically distributed random variables independent of $\left(Y_{i}\right)$ with $\mathrm{E}[Y]<\mathrm{E}[T]$, then the ladder height distribution is tail-equivalent to $G_{0}$. Because the geometric distribution of the number of ladder heights is light-tailed, the tail of the distribution of the maximum of the random walk can be obtained from Theorem 1(i) provided $G_{0} \in \mathscr{S}$. This is why Asmussen et al. (1999a) need the assumption $F_{0} \in \mathscr{S}$ in some of their results.

In Klüppelberg (1988) a class $\mathscr{S}^{*}$ of distributions is introduced via the definition $G \in \mathscr{S}^{*}$ if and only if $\int_{0}^{\infty} \bar{G}(y) \mathrm{d} y<\infty$ and

$$
\lim _{x \rightarrow \infty} \int_{0}^{x} \frac{\bar{G}(x-y)}{\bar{G}(x)} \bar{G}(y) \mathrm{d} y=2 \int_{0}^{\infty} \bar{G}(y) \mathrm{d} y .
$$

The use of L'Hôpital's rule verifies that $G_{0} \in \mathscr{S}$ if $G \in \mathscr{S}^{*}$. This motivates the definition of $\mathscr{S}^{*}$. Moreover, $G \in \mathscr{S}^{*}$ implies $G \in \mathscr{S}$; see Klüppelberg (1988). It should be noticed that $G \in \mathscr{S}^{*}$ implies that the density of $G_{0}$ is a subexponential density. A subexponential density is a density $g$ with $g(x+y) / g(x) \rightarrow 1$ for all $y$ and $g * g(x) / g(x) \rightarrow 2$ as $x \rightarrow \infty$. Thus analogues of Theorems 1 and 2 are also possible for subexponential densities.

Obviously $\mathscr{R} \subset \mathscr{S}^{*}$. Note that it is not possible to interchange limit and integral in the above definition. We obtain the following result. 


\section{Theorem 2.}

(i) Assume that there exists an $\varepsilon>0$ such that $E\left[(1+\varepsilon)^{N}\right]<\infty$. If $Y \in \mathscr{S}^{*}$ then $S_{N} \in \mathscr{S}^{*}$.

(ii) Assume that $G \in \Gamma$ and that $\mathrm{E}[Y-x \mid Y>x] \leqslant B<\infty$ for all $x$ such that $P[Y>x]>0$ and for some $B \in \mathbb{R}$. If $N \in \mathscr{S}^{*}$ then $S_{N} \in \mathscr{S}^{*}$.

Corollary 2. Let $N$ have a mixed Poisson distribution. If $H \in \mathscr{S}^{*}$ then $N \in \mathscr{S}^{*}$. If, moreover, $G \in \Gamma$ and for some $B \in \mathbb{R}, \mathrm{E}[Y-x \mid Y>x] \leqslant B<\infty$ for all $x$ such that $P[Y>x]>0$, then $S_{N} \in \mathscr{S}^{*}$.

The condition $\mathrm{E}[Y-x \mid Y>x] \leqslant B$ is also a light-tail assumption. For a discussion of this assumption, see Hogg and Klugman (1984) or Embrechts et al. (1993).

The conditions $G \in \Gamma$ and $\mathrm{E}[Y-x \mid Y>x] \leqslant B$ seem to be far from necessary, as the results of Grandell (1997) for $N \in \mathscr{R}$ indicate. But these conditions appear in a natural way from the proofs below. The author conjectures that there exist counterexamples if the conditions are violated. In order to construct a counterexample, the asymptotic behaviour of the tail of $F$ would be useful to know. But this cannot be obtained by our approach.

\section{Proofs of the results}

We start by recalling some properties of subexponential distributions. The proofs can be found in Athreya and Ney (1972) and Chistyakov (1964) or are straightforward.

\section{Lemma 2.}

(i) If

$$
\varlimsup_{x \rightarrow \infty} \frac{\overline{G^{* 2}}(x)}{\bar{G}(x)} \leqslant 2
$$

then $G \in \mathscr{S}$.

(ii) If

$$
\varlimsup_{x \rightarrow \infty} \int_{0}^{x} \frac{\bar{G}(x-y)}{\bar{G}(x)} \bar{G}(y) \mathrm{d} y \leqslant 2 \int_{0}^{\infty} \bar{G}(y) \mathrm{d} y
$$

then $G \in \mathscr{S}^{*}$.

(iii) If $G \in \mathscr{S}$ then for any $\varepsilon>0$ there exists a $D \in \mathbb{R}$ (independent of $n$ ) such that $\bar{G}^{* n}(x) \leqslant D(1+\varepsilon)^{n} \bar{G}(x)$ for all $n \in \mathbb{N}$ and all $x \geqslant 0$. Moreover,

$$
\lim _{x \rightarrow \infty} \frac{\overline{G^{* n}}(x)}{\bar{G}(x)}=n .
$$

(iv) Assume that $\overline{G^{\prime}}(x) / \bar{G}(x) \rightarrow c \in(0, \infty)$. Then $G^{\prime} \in \mathscr{S}$ if and only if $G \in \mathscr{S}$.

(v) Let $G \in \mathscr{S}$. Then for any $y \in \mathbb{R}$ 


$$
\lim _{x \rightarrow \infty} \frac{\bar{G}(x-y)}{\bar{G}(x)}=1
$$

uniformly for y-compact sets.

(vi) Let $G \in \mathscr{S}$. For any $\varepsilon>0$ one has $\bar{G}(x) \mathrm{e}^{\varepsilon x} \rightarrow \infty$ as $x \rightarrow \infty$. Equivalently, for any $\varepsilon>0$ there exists a $c=c(\varepsilon)>0$ such that $\bar{G}(x)>c \mathrm{e}^{-\varepsilon x}$.

(vii) Let $G \in \mathscr{S}$ and $G^{\prime}$ be a distribution function such that $\overline{G^{\prime}}(x) / \bar{G}(x) \rightarrow 0$. Then $\overline{G * G^{\prime}}(x) / \bar{G}(x) \rightarrow 1$.

Proof of Lemma 1. We prove by induction that

$$
\lim _{x \rightarrow \infty} \frac{\overline{G^{*(m+1)}}(x)}{\overline{G^{* m}}(x)}=\infty .
$$

Assume $\overline{G^{* m}}(x) / \overline{G^{*(m-1)}}(x) \rightarrow \infty$ and let $C>0$. There exists $x_{0}$ such that for all $x \geqslant x_{0}$ one has $\overline{G^{* m}}(x) / \overline{G^{*(m-1)}}(x) \geqslant C$. Then

$$
\begin{aligned}
\frac{\overline{G^{*(m+1)}}(x)}{\overline{G^{* m}}(x)} & \geqslant \frac{1}{\overline{G^{* m}}(x)} \int_{0}^{x} \frac{\overline{G^{* m}}(x-y)}{\overline{G^{*(m-1)}}(x-y)} \overline{G^{*(m-1)}}(x-y) \mathrm{d} G(y) \\
& \geqslant \frac{1}{\overline{G^{* m}}(x)} C \int_{0}^{x-x_{0}} \overline{G^{*(m-1)}}(x-y) \mathrm{d} G(y) \geqslant C\left(1-\frac{\bar{G}\left(x-x_{0}\right)}{\overline{G^{* m}}(x)}\right) .
\end{aligned}
$$

Using $\overline{G^{* m}}(x) \geqslant \overline{G^{* 2}}(x)$, it follows that

$$
\varliminf_{x \rightarrow \infty} \frac{\overline{G^{*(m+1)}}(x)}{\overline{G^{* m}}(x)} \geqslant C(1-b) .
$$

Because $C$ is arbitrary the result is proved.

We prove now Theorem 1. The proof of (i) can be found in Grandell (1997) or Athreya and Ney (1972, p. 150).

For the proof of (ii) we also need to express $P[S>x]$ in terms of the tail probabilities $P[N>n]$.

Lemma 3. The tail probability of $S_{N}$ can be represented as

$$
P\left[S_{N}>x\right]=\sum_{n=0}^{\infty} P[N>n]\left(G^{* n}(x)-G^{*(n+1)}(x)\right) .
$$

Proof. This follows readily from

$$
P\left[S_{N}>x\right]=\sum_{m=1}^{\infty} p_{m} \overline{G^{* m}}(x)=\sum_{m=1}^{\infty} p_{m} \sum_{n=0}^{m-1}\left(G^{* n}(x)-G^{*(n+1)}(x)\right) .
$$


Proof of Theorem 1(ii). We have to consider

$$
\frac{P\left[\sum_{i=1}^{N_{1}+N_{2}} Y_{i}>x\right]}{P\left[\sum_{i=1}^{N} Y_{i}>x\right]}=\frac{\left.\left.\sum_{n=0}^{\infty} P\left[N_{1}+N_{2}>n\right]\right) G^{* n}(x)-G^{*(n+1)}(x)\right)}{\sum_{n=0}^{\infty} P[N>n]\left(G^{* n}(x)-G^{*(n+1)}(x)\right)} .
$$

We have to show that the first few terms do not matter. Let $\varepsilon>0$. Then there exists an $M \in \mathbb{N}$ such that $P\left[N_{1}+N_{2}>n\right]<(2+\varepsilon) P[N>n]$ for all $n \geqslant M$. For the first $M$ terms

$$
\begin{aligned}
\sum_{n=0}^{M-1} P\left[N_{1}+N_{2}>n\right]\left(G^{* n}(x)-G^{*(n+1)}(x)\right) & \leqslant \sum_{n=0}^{M-1}\left(G^{* n}(x)-G^{*(n+1)}(x)\right) \\
& =\overline{G^{* M}(x)} .
\end{aligned}
$$

If $G\left(x_{0}\right)=1$ for some $x_{0}$ then obviously, for $x \geqslant M x_{0}$,

$$
\frac{\overline{G^{* M}(x)}}{\sum_{n=1}^{\infty} p_{n} \overline{G^{* n}}(x)}=0,
$$

because for any $n_{0} \in \mathbb{N}$ there exists $n>n_{0}$ with $p_{n}>0$. Assume therefore that $G(x)<1$ for all $x \in \mathbb{R}$. Then

$$
\frac{\overline{G^{* M}(x)}}{\sum_{n=1}^{\infty} p_{n} \overline{G^{* n}}(x)} \leqslant \frac{1}{\sum_{n=M}^{\infty} p_{n} \overline{G^{* n}}(x) / \overline{G^{* M}}(x)}
$$

But because by Lemma 2(vi), for $a>1$,

$$
\sum_{n=0}^{\infty} p_{n} a^{n}=\mathrm{E}\left[a^{N}\right]=\infty,
$$

it follows from (2) and Fatou's lemma that

$$
\frac{\overline{G^{* M}(x)}}{\sum_{n=1}^{\infty} p_{n} \overline{G^{* n}}(x)} \rightarrow 0 \quad \text { as } x \rightarrow \infty .
$$

For the remaining term we find

$$
\begin{aligned}
\sum_{n=M}^{\infty} P\left[N_{1}+N_{2}>n\right]\left(G^{* n}(x)-G^{*(n+1)}(x)\right) & \leqslant(2+\varepsilon) \sum_{n=M}^{\infty} P[N>n]\left(G^{* n}(x)-G^{*(n+1)}(x)\right) \\
& \leqslant(2+\varepsilon) \sum_{n=0}^{\infty} P[N>n]\left(G^{* n}(x)-G^{*(n+1)}(x)\right) .
\end{aligned}
$$


It follows that

$$
\varlimsup_{n \rightarrow \infty} \frac{P\left[\sum_{i=1}^{N_{1}+N_{2}} Y_{i}>x\right]}{P\left[\sum_{i=1}^{N} Y_{i}>x\right]} \leqslant 2+\varepsilon .
$$

Because $\varepsilon$ was arbitrary, the assertion follows.

For the mixed Poisson case we need the following representation from Grandell (1997), which is easy to prove.

Lemma 4. Let $N$ have a mixed Poisson distribution. Then

$$
P[N>n]=\int_{0}^{\infty} \frac{x^{n}}{n !} \mathrm{e}^{-x} \bar{H}(x) \mathrm{d} x .
$$

Proof of Corollary 1. Note that $N_{1}+N_{2}$ has the same distribution as a mixed Poisson distribution with mixing distribution $H^{* 2}$. Thus

$$
\frac{P\left[N_{1}+N_{2}>n\right]}{P\left[N_{1}>n\right]}=\frac{\int_{0}^{\infty}\left(x^{n} / n !\right) \mathrm{e}^{-x} \overline{H^{* 2}}(x) \mathrm{d} x}{\int_{0}^{\infty}\left(x^{n} / n !\right) \mathrm{e}^{-x} \bar{H}(x) \mathrm{d} x} .
$$

Choose $\varepsilon>0$. There exists an $\ell_{0}$ such that

$$
\frac{\overline{H^{* 2}}(x)}{\bar{H}(x)} \leqslant 2+\varepsilon \quad \text { for all } x \geqslant \ell_{0} .
$$

Intuitively $N$ can only become large if the Poisson parameter is large, in particular larger than $\ell_{0}$. Indeed, for $c=\inf \left\{\mathrm{e}^{x} \bar{H}(x): x \geqslant 0\right\}>0$ (see Lemma 2(vi))

$$
\frac{\int_{0}^{\ell_{0}}\left(x^{n} / n !\right) \mathrm{e}^{-x} \overline{H^{* 2}}(x) \mathrm{d} x}{\int_{0}^{\infty}\left(x^{n} / n !\right) \mathrm{e}^{-x} \bar{H}(x) \mathrm{d} x} \leqslant \frac{\int_{0}^{\ell_{0}} x^{n} \mathrm{e}^{-x} \mathrm{~d} x}{\int_{0}^{\infty} x^{n} \mathrm{e}^{-x} c \mathrm{e}^{-x} \mathrm{~d} x}=\frac{2}{c} \int_{0}^{\ell_{0}} \frac{(2 x)^{n}}{n !} \mathrm{e}^{-x} \mathrm{~d} x,
$$

which is readily seen to converge to 0 as $n \rightarrow \infty$. The remainder can be estimated as

$$
\frac{\int_{\ell_{0}}^{\infty}\left(x^{n} / n !\right) \mathrm{e}^{-x} \overline{H^{* 2}}(x) \mathrm{d} x}{\int_{0}^{\infty}\left(x^{n} / n !\right) \mathrm{e}^{-x} \bar{H}(x) \mathrm{d} x} \leqslant(2+\varepsilon) \frac{\int_{\ell_{0}}^{\infty} x^{n} \mathrm{e}^{-x} \bar{H}(x) \mathrm{d} x}{\int_{0}^{\infty} x^{n} \mathrm{e}^{-x} \bar{H}(x) \mathrm{d} x} \leqslant 2+\varepsilon .
$$

It follows that 


$$
\varlimsup_{n \rightarrow \infty} \frac{P\left[N_{1}+N_{2}>n\right]}{P\left[N_{1}>n\right]} \leqslant 2+\varepsilon
$$

Because $\varepsilon$ was arbitrary, the assertion follows.

We now start with the proof of Theorem 2 .

Proof of Theorem 2(i). Note that $\mathrm{E}[N]<\infty$ and $\mathrm{E}\left[S_{N}\right]=\mathrm{E}[N] \mathrm{E}[Y]<\infty$. Since $Y \in$ $\mathscr{S}^{*} \subset \mathscr{S}$ we have $S_{N} \in \mathscr{S}$. Recall from Theorem 1(i) that $P\left[S_{N}>x\right] \sim \mathrm{E}[N] P[Y>x]$. The assertion follows now from Lemma 2(v) and Theorem 2.1(b) of Klüppelberg (1988).

The second statement turns out to be the hardest one to prove. This is also indicated by the additional condition needed. We start by proving two lemmas.

Lemma 5. Under the conditions of Theorem 1(ii),

$$
\lim _{x \rightarrow \infty} P\left[S_{N} \leqslant x \mid S_{N}+Y_{N+1}>x\right]=0 .
$$

Under the conditions of Theorem 2(ii) one has

$$
\lim _{x \rightarrow \infty} \frac{\mathrm{E}\left[\left(S_{N}+Y_{N+1}-x\right) \llbracket\left(\mathrm{S}_{N}<x<S_{N}+Y_{N+1}\right)\right]}{P\left[S_{N}>x\right]}=0 .
$$

Proof. Recall from the proof of Theorem 1(ii) that $\bar{G}(x) / \bar{F}(x) \rightarrow 0$ as $x \rightarrow \infty$. The first assertion then follows from Lemma 2(vii), noting that $P\left[S_{N} \leqslant x<S_{N}+Y_{N+1}\right] /$ $P\left[S_{N}+Y_{N+1}>x\right]=1-P\left[S_{N}>x\right] / P\left[S_{N}+Y_{N+1}>x\right]$. To prove the second assertion we note that

$$
\begin{aligned}
\mathrm{E}\left[\mathrm{E}\left[Y_{N+1}-\left(x-S_{N}\right) \mid S_{N}, Y_{N+1}>\left(x-S_{N}\right)\right]\right]\left(S_{N}<x<S_{N}\right. & \left.\left.+Y_{N+1}\right)\right] \\
& \leqslant B P\left[S_{N}<x<S_{N}+Y_{N+1}\right],
\end{aligned}
$$

and now the assertion follows from the first part and Lemma 2(vii), recalling that $S_{N} \in \mathscr{S}$.

Lemma 6. Let $G$ be a distribution with $G(0)=0$ and $\int_{0}^{\infty} \bar{G}(y) \mathrm{d} y<\infty$, and let $S_{n}$ have distribution function $G^{* n}$. Then

$$
\begin{aligned}
& \int_{0}^{x}\left(G^{* m}(x-y)-G^{*(m+1)}(x-y)\right)\left(G^{*(n-m)}(y)-G^{*(n-m+1)}(y)\right) \mathrm{d} y \\
& =\mathrm{E}[Y] P\left[S_{n}<x \leqslant S_{n+1}\right]+\mathrm{E}\left[\left(S_{n+2}-x\right) \llbracket\left(S_{n+1}<x<S_{n+2}\right)\right]-E\left[\left(S_{n+1}-x\right) \llbracket\left(S_{n}<x<S_{n+1}\right)\right] .
\end{aligned}
$$

In particular, the expression is independent of $m$.

Proof. The independence of $m$ follows readily by calculating the Laplace transform. Suppose, therefore, that $m=0$. We write $S_{n}=\sum_{i=1}^{n} Y_{i}$. Then 


$$
\begin{aligned}
& \int_{0}^{x} \bar{G}(x-y)\left(G^{* n}(y)-G^{*(n+1)}(y)\right) \mathrm{d} y \\
&=\mathrm{E}\left[\int_{0}^{x}\left\{\left(y>x-Y_{n+2}\right) \llbracket\left(S_{n} \leqslant y<S_{n+1}\right) \mathrm{d} y\right]\right. \\
&=\mathrm{E}\left[\left(\left\{S_{n+1} \wedge x\right\}-\left\{S_{n} \vee x-Y_{n+2}\right\}\right) \llbracket\left(S_{n}<x<S_{n+2}\right)\right] \\
&=\mathrm{E}\left[\left(\left\{\left(S_{n+2}-x\right) \wedge Y_{n+2}\right\}-\left\{S_{n}+Y_{n+2}-x\right\}^{+}\right) \llbracket\left(S_{n}<x<S_{n+2}\right)\right] \\
&=\mathrm{E}\left[\left(\left\{\left(S_{n+2}-x\right) \wedge Y_{n+2}\right\}-\left\{S_{n+1}-x\right\}^{+}\right) \llbracket\left(S_{n}<x<S_{n+2}\right)\right] \\
&=\mathrm{E}\left[Y_{n+2}\right] P\left[S_{n}<x \leqslant S_{n+1}\right]-\mathrm{E}\left(\left[S_{n+1}-x\right) \llbracket\left(S_{n}<x<S_{n+1}\right)\right] \\
&+\mathrm{E}\left[\left(S_{n+2}-x\right) \llbracket\left(S_{n+1}<x<S_{n+2}\right)\right] .
\end{aligned}
$$

Note that the condition $N \in \mathscr{S}^{*}$ can be written as $\mathrm{E}[N]<\infty$ and

$$
\lim _{n \rightarrow \infty} \sum_{m=0}^{n} \frac{P[N>m] P[N>n-m]}{P[N>n]}=2 \mathrm{E}[N] .
$$

Proof of Theorem 2(ii). Note that $\mathrm{E}[Y] \leqslant B$ and $\mathrm{E}\left[S_{N}\right]=\mathrm{E}[N] \mathrm{E}[Y]<\infty$. By Lemma 3 we can write

$$
\begin{aligned}
& \int_{0}^{x} P\left[S_{N}>x-y\right] P\left[S_{N}>y\right] \mathrm{d} y \\
& =\sum_{m=0}^{\infty} \sum_{k=0}^{\infty} P[N>m] P[N>k] \int_{0}^{x}\left(G^{* m}(x-y)-G^{*(m+1)}(x-y)\right)\left(G^{* k}(y)-G^{*(k+1)}(y)\right) \mathrm{d} y \\
& =\sum_{n=0}^{\infty} \sum_{m=0}^{n} P[N>m] P[N>n-m] \int_{0}^{x}\left(G^{* m}(x-y)-G^{*(m+1)}(x-y)\right)\left(G^{*(n-m)}(y)\right. \\
& \left.\quad-G^{*(n-m+1)}(y)\right) \mathrm{d} y
\end{aligned}
$$

Choose $\varepsilon>0$. There exists a $M$ such that, for all $n \geqslant M$,

$$
\sum_{m=0}^{n} \frac{P[N>m] P[N>n-m]}{P[N>n]} \leqslant 2 \mathrm{E}[N]+\varepsilon .
$$

Then 


$$
\begin{aligned}
& \sum_{n=0}^{M-1} \sum_{m=0}^{n} P[N>m] P[N>n-m]\left(\mathrm{E}[Y] P\left[S_{n}<x \leqslant S_{n+1}\right]\right. \\
& \left.+\mathrm{E}\left[\left(S_{n+2}-x\right) \llbracket\left(\mathrm{S}_{n+1}<x<S_{n+2}\right)\right]-\mathrm{E}\left[\left(S_{n+1}-x\right) \llbracket\left(\mathrm{S}_{n}<x<S_{n+1}\right)\right]\right) \\
& \leqslant M \sum_{n=0}^{M-1} \mathrm{E}[Y] P\left[S_{n}<x \leqslant S_{n+1}\right]+\mathrm{E}\left[\left(S_{n+2}-x\right) \llbracket\left(\mathrm{S}_{n+1}<x<S_{n+2}\right)\right] \\
& \quad-\mathrm{E}\left[\left(S_{n+1}-x\right) \llbracket\left(\mathrm{S}_{n}<x<S_{n+1}\right)\right] \\
& \leqslant M(\mathrm{E}[Y]+B) \overline{G^{*(M+1)}}(x-) .
\end{aligned}
$$

Using Lemma 6, it now follows as in the proof of Theorem 1(ii) that the above term has no asymptotic contribution.

Because of Lemma 6, it remains to show that

$$
\begin{gathered}
\sum_{n=0}^{\infty} P[N>n]\left(\mathrm{E}[Y] P\left[S_{n}<x \leqslant S_{n+1}\right]+\mathrm{E}\left[\left(S_{n+2}-x\right) \rrbracket\left(\mathrm{S}_{n+1}<x<S_{n+2}\right)\right]\right. \\
\left.-\mathrm{E}\left[\left(S_{n+1}-x\right) \llbracket\left(\mathrm{S}_{n}<x<S_{n+1}\right)\right]\right) / P\left[S_{N}>x\right] \rightarrow \mathrm{E}[Y]
\end{gathered}
$$

as $x \rightarrow \infty$ or, equivalently,

$$
\begin{aligned}
\sum_{n=0}^{\infty} P & {[N>n]\left(\mathrm{E}\left[\left(S_{n+2}-x\right) \llbracket\left(\mathrm{S}_{n+1}<x<S_{n+2}\right)\right]\right.} \\
& \left.-\mathrm{E}\left[\left(S_{n+1}-x\right) \llbracket\left(\mathrm{S}_{n}<x<S_{n+1}\right)\right]\right) / P\left[S_{N}>x\right] \rightarrow 0 .
\end{aligned}
$$

It follows readily that the left-hand side can be written as

$$
\left(\mathrm{E}\left[\left(S_{N}+Y_{N+1}-x\right) \llbracket\left(\mathrm{S}_{N}<x<S_{N}+Y_{N+1}\right)\right]-\mathrm{E}\left[\left(Y_{1}-x\right) \llbracket\left(Y_{1}>x\right)\right]\right) / P\left[S_{N}>x\right] .
$$

In view of Lemma 5, this proves the theorem.

The next proof is similar to the proof of Corollary 1 .

Proof of Corollary 2. Note that $\mathrm{E}[N]=\int_{0}^{\infty} \bar{H}(x) \mathrm{d} x<\infty$. Using Lemma 4, we find

$$
\begin{aligned}
\sum_{m=0}^{n} P[N>m] P[N>n-m] & =\int_{0}^{\infty} \int_{0}^{\infty} \sum_{m=0}^{n} \frac{x^{m} y^{n-m}}{m !(n-m) !} \mathrm{e}^{-(x+y)} \bar{H}(x) \bar{H}(y) \mathrm{d} y \mathrm{~d} x \\
& =\int_{0}^{\infty} \int_{0}^{\infty} \frac{(x+y)^{n}}{n !} \mathrm{e}^{-(x+y)} \bar{H}(x) \bar{H}(y) \mathrm{d} y \mathrm{~d} x \\
& =\int_{0}^{\infty} \int_{0}^{z} \frac{z^{n}}{n !} \mathrm{e}^{-z} \bar{H}(x) \bar{H}(z-x) \mathrm{d} x \mathrm{~d} z .
\end{aligned}
$$

Let $\varepsilon>0$. Choose $\ell_{0}$ such that 


$$
\int_{0}^{z} \bar{H}(x) \bar{H}(z-x) \mathrm{d} x \leqslant\left(2 \int_{0}^{\infty} \bar{H}(x) \mathrm{d} x+\varepsilon\right) \bar{H}(z)
$$

for all $z \geqslant \ell_{0}$. It follows as in the proof of Corollary 1 that

$$
\lim _{x \rightarrow \infty} \frac{\int_{0}^{\ell_{0}} \int_{0}^{z}\left(z^{n} / n !\right) \mathrm{e}^{-z} \bar{H}(x) \bar{H}(z-x) \mathrm{d} x \mathrm{~d} z}{\int_{0}^{\infty}\left(z^{n} / n !\right) \mathrm{e}^{-z} \bar{H}(z) \mathrm{d} z} \leqslant \lim _{x \rightarrow \infty} \frac{\int_{0}^{\ell_{0}} z^{n+1} \mathrm{e}^{-z} \mathrm{~d} z}{\int_{0}^{\infty} z^{n} \mathrm{e}^{-z} \bar{H}(z) \mathrm{d} z}=0 .
$$

The rest of the proof now proceeds similarly to the proof of Corollary 1 .

\section{An example}

Let $\left(\left(L_{i}, \sigma_{i}\right)\right)$ be a sequence of independent identically distributed vectors with $L_{i} \geqslant 0$ and $0<\sigma_{i} \leqslant s_{0}$ almost surely. Set $\Sigma_{n}=\sum_{i=1}^{n} \sigma_{i}$. The times $\Sigma_{i}$ are the times where the intensity changes. Let $\lambda_{t}=L_{n}$ if $\Sigma_{n-1}<t \leqslant \Sigma_{n}$ denote the intensity at time $t$ and let $\Lambda_{t}=\int_{0}^{t} \lambda_{s} \mathrm{~d} s$. Finally, let $N(t)=\tilde{N}\left(\Lambda_{t}\right)$ be a point process where $(\hat{N}(t))$ is a homogeneous Poisson process with rate 1 . As before, the sequence $\left(Y_{i}\right)$ of positive independent and identically distributed random variables is assumed to be independent of $(N(t))$. The stochastic process

$$
X(t)=\sum_{i=1}^{N(t)} Y_{i}-t
$$

is a special case of a Björk and Grandell (1988) model. If $\sigma_{i}=s_{0}$ is deterministic it is the Ammeter (1948) model; see also Grandell (1995). We are interested in the tail of the maximum $M=\sup \{X(t): t \geqslant 0\}$ of the stochastic process $(X(t))$. This tail probability is called the ruin probability in risk theory, the steady-state waiting time in queuing theory and the stationary dam content in storage theory. In order that $M \neq \infty$ almost surely we have to assume that $\mathrm{E}[L \sigma] \mathrm{E}[Y]<\mathrm{E}[\sigma]$. This can be interpreted as saying that in an interval of length $\sigma$ the mean income (or outflow) is larger than the mean expenditure (input).

In Grandell (1995) the tail probability of $M$ is investigated in the case of an Ammeter model and summands $Y$ such that the integrated tail distribution

$$
G_{0}(x)=\frac{1}{\mathrm{E}[Y]} \int_{0}^{x} \bar{G}(y) \mathrm{d} y \in \mathscr{S} .
$$

This is, for instance, the case if $G \in \mathscr{S}^{*}$. In Asmussen et al. (1999a) the assertion is shown for a general Björk-Grandell model. We treat here the case of subexponential intensity levels.

Theorem 3. Let $(X(t))$ be a Björk-Grandell model with $\sigma_{i} \leqslant s_{0}$. Denote the marginal distribution of $L_{i}$ by $H$. Then under the conditions of Corollary 2 we have

$$
\lim _{x \rightarrow \infty} \frac{P[M>x]}{\overline{F_{0}}(x)}=\frac{\mathrm{E}[L \sigma] \mathrm{E}[Y]}{\mathrm{E}[\sigma]-\mathrm{E}[L \sigma] \mathrm{E}[Y]},
$$


where $F$ is the distribution of $X_{\sigma_{1}}$.

Proof. The theorem would follow immediately from Theorem 2 and Embrechts and Veraverbeke (1982) if we could replace $M$ by $M^{*}=\sup \left\{X\left(\Sigma_{n}\right): n \in \mathbb{N}\right\}$. It is therefore enough to show that $P\left[M^{*}>x\right] / P[M>x] \rightarrow 1$ as $x \rightarrow \infty$. It is clear that $P\left[M^{*}>x\right]<P[M>x]$ and $P\left[M^{*}>x-s_{0}\right]>P[M>x]$. From Theorem 2 it follows that $F \in \mathscr{S}^{*}$ and therefore $F_{0} \in \mathscr{S}$. The theorem now follows because

$$
\lim _{x \rightarrow \infty} \frac{\bar{F}_{0}(x)}{\bar{F}_{0}\left(x-s_{0}\right)}=1
$$

by Lemma $2(\mathrm{v})$.

The above theorem shows the limits of the application of Theorems 1 and 2. Because no explicit tail behaviour for $S_{N}$ was obtained, one has to use $F_{0}$. The problem of finding the asymptotic behaviour of $\overline{F_{0}}(x)$ if $F \notin \mathscr{B}$ seems to be hard. For $N \in \mathscr{B}$ one can show that

$$
P\left[S_{N}>x \mathrm{E}[Y]\right] \sim P[N>x] .
$$

This yields another open question. When does (5) hold? Some special cases of this problem are considered in Asmussen et al. (1999b).

\section{Acknowledgements}

Various useful remarks by Jan Grandell and an unknown referee are acknowledged, which in particular led to a simplification of the proof of Theorem 2. The author also thanks Søren Asmussen for pointing the author's attention to the counterexample from Asmussen et al. (1999b).

\section{References}

Ammeter, H. (1948) A generalization of the collective theory of risk in regard to fluctuating basic probabilities. Skand. Aktuar. Tidskr, 31, 171-198.

Asmussen, S., Schmidli, H. and Schmidt, V. (1999a) Tail probabilities for non-standard risk and queuing processes with subexponential jumps. Adv. in Appl. Probab., 31. To appear.

Asmussen, S., Klüppelberg, C. and Sigman, K. (1999b) Sampling at subexponential times, with queuing applications. Stochastic Process. Appl., 79, 265-286.

Athreya, K.B. and Ney, P. (1972) Branching Processes. Berlin: Springer-Verlag.

Björk, T. and Grandell, J. (1988) Exponential inequalities for ruin probabilities in the Cox case. Scand. Actuar. J., 77-111.

Chistyakov, V.P. (1964) A theorem on sums of independent, positive random variables and its applications to branching processes. Theory Probab. Appl., 9, 640-648.

Embrechts, P. and Goldie, C.M. (1982) On convolution tails. Stochastic Process. Appl., 13, 263-278.

Embrechts, P. and Veraverbeke, N. (1982) Estimates for the probability of ruin with special emphasis on the possibility of large claims. Insurance Math. Econom., 1, 55-72. 
Embrechts, P., Grandell, J. and Schmidli, H. (1993) Finite-time Lundberg inequalities in the Cox case. Scand. Actuar. J., 17-41.

Grandell, J. (1991) Aspects of Risk Theory. New York: Springer-Verlag.

Grandell, J. (1995) Some remarks on the Ammeter risk process. Schweiz. Verein. Versicherungsmath. Mitt., 95, 43-71.

Grandell, J. (1997) Mixed Poisson Processes. London: Chapman \& Hall.

Hogg, R.V. and Klugman, S.A. (1984) Loss Distributions. New York: Wiley.

Klüppelberg, C. (1988) Subexponential distributions and integrated tails. J. Appl. Probab., 25, 132141.

Schmidli, H. (1996) Lundberg inequalities for a Cox model with a piecewise constant intensity. $J$. Appl. Probab., 33, 196-210.

Stam, A.J. (1973) Regular variation of the tail of a subordinated probability distribution. Adv. Appl. Probab., 5, 308-327.

Veraverbeke, N. (1977) Asymptotic behaviour of Wiener-Hopf factors of a random walk. Stochastic Process. Appl., 5, 27-37.

Received June 1997 and revised April 1998 